\title{
Blow Up of Incompressible Euler Solutions *
}

\author{
JOHAN HOFFMAN ${ }^{1}$ and CLAES JOHNSON ${ }^{1}$
}

\author{
April 12008 \\ ${ }^{1}$ School of Computer Science and Communication \\ Royal Institute of Technology \\ 10044 Stockholm, Sweden. \\ email: jhoffman@csc.kth.se, cgjoh@csc.kth.se
}

\begin{abstract}
.
We present analytical and computational evidence of blowup of initially smooth solutions of the incompressible Euler equations into non-smooth turbulent solutions. We detect blowup by observing increasing $L_{2}$-residuals of computed solutions under decreasing mesh size.
\end{abstract}

AMS subject classification (2000): 35Q30, 65M60.

\section{The Clay Navier-Stokes Millennium Problem}

The Clay Mathematics Institute Millennium Problem on the incompressible Navier-Stokes equations formulated by Fefferman [7] asks for a proof of (I) global existence of smooth solutions for all smooth data, or a proof of the converse (II) non global existence of a smooth solution for some smooth data, referred to as breakdown or blowup. A proof of (I) may have to be analytical to cover all smooth data, while it is conceivable that the converse (II) can be proved by computation for a specific choice of smooth data.

In this note we address the analogous problem for the inviscid incompressible Euler equations, which for some reason is not explicitely a Millennium Problem, although mentioned briefly in [7] and in [6] referred to as "a major open problem in PDE theory, of far greater physical importance than the blowup problem for Navier-Stokes equations, which of course is known to the nonspecialists because it is a Clay Millenium Problem". In fact, since the viscosity in the Navier-Stokes equations is allowed to be arbitarily small and solutions of the Euler equations are defined as viscosity solutions of the Navier-Stokes equations under vanishing viscosity, the Euler equations effectively are included in the Millenium Problem.

We present evidence that a specific initially smooth solution of the Euler equations, potential flow around a circular cylinder, in finite time exhibits blowup into a turbulent non-smooth solution, that is we present evidence of (II). More

\footnotetext{
*This article is dedicated by the second author to Juhani Pitkäranta in memory of three decades of fruitful interaction (without blowup).
} 
generally, we present evidence that all (non-trivial) initially smooth Euler solutions exhibit blowup into turbulent solutions. This work closely connects to the new resolution of d'Alembert's paradox presented in [15].

We solve the Euler equations computationally using a least squares residualstabilized finite element method referred to as EG2 as an acronym of Euler General Galerkin. We shall see that EG2 is not just any ad hoc computational method but captures fundamentals.

We use an objective operational definition of non smooth solution of the Euler equations as a viscosity solution with substantial effect of vanishing viscosity in the form of substantial turbulent viscous dissipation, which characterizes a turbulent solution. We find that an initially smooth EG2 solution becomes turbulent and thus shows blowup into a non-smooth solution. We present evidence that blowup detection is stable under infinite mesh refinement/vanishing viscosity, so that detection of blowup of a EG2 solution on a mesh of finite mesh size can be used as detection of blowup under vanishing viscosity.

Since our evidence is critically based on computation, we are led to the general question of the significance of computational solutions to differential equations. A basic question is what quality requirements should be put on computed solutions of differential equations to allow them to replace exact solutions. We shall see that an affirmative answer can be given in terms of Hadamard's notion of wellposedness [9] and residual-based a posteriori error estimation [13]. We first recall some basic facts of general nature and we then turn to the Euler blowup problem, starting with compressible flow and then addressing the Millennium problem of incompressible flow under vanishing viscosity.

\section{Existence of Exact Solution from Computed Solution}

Consider an equation $A(x)=b$, where $A: \mathbb{R} \rightarrow \mathbb{R}$ is a continuously differentiable function with derivative $A^{\prime}(x)$ and $b \in \mathbb{R}$ is a given real number. Assume that for some $X \in \mathbb{R}$ and some (small) positive error tolerance $T O L$,

$$
2 S|R(X)|<T O L
$$

where $R(X)=A(X)-b$ is the residual of $X, S=\left|A^{\prime}(X)^{-1}\right|$ is a stability factor and $S\left|A^{\prime}(y)-A^{\prime}(X)\right|<\frac{1}{2}$ for $|y-X|<T O L$. Then there is $x \in \mathbb{R}$ with $|x-X|<T O L$ such that $A(x)=b$. This follows from the contraction mapping principle applied to the mapping $y \rightarrow y-A^{\prime}(X)^{-1}(A(y)-b)$, and can be generalized to Banach spaces.

The existence of a computed approximate solution $X$ with sufficiently small residual $R(X)$ modulo the stability factor $S$, thus guarantees the existence of an exact solution $x$ with vanishing residual $R(x)=0$ within distance $2 S R$. A result of this nature for the Navier-Stokes equations is given in $[2,4]$.

The (potentially immense) advantage of a computed solution is that it is available for inspection, while an exact root only known to exist (e.g. by contraction mapping) in general cannot be inspected. For example, the computation $2-1.4^{2}=0.04$, shows that $\sqrt{2} \approx 1.4$ with an error less than $(2.8)^{-1} 0.04<0.015$, while inspecting the mere symbol $\sqrt{2}$ reveals nothing about its value. 


\section{Wellposedness}

Since Hadamard [9] it is well understood that solving (differential) equations, perturbations of data have to be taken into account. If a vanishingly small perturbation can have a major effect on a solution, then the solution (or problem) is illposed, and in this case the solution may not carry any meaningful information and thus may be meaningless from both mathematical and applications points of view. According to Hadamard, only a wellposed solution, for which small perturbations have small effects (in some suitable sense), can be meaningful. In this perspective it is remarkable that wellposedness is not an issue in the Millenium Problem formulation [7]. However, Fefferman wants to give "reasonable lee-way (in the problem formulation) to solvers while retaining the heart of the problem", and we use this lee-way to bring wellposedness into the heart of a meaningful discussion of the Millennium Problem.

Perturbations of the data $b$ of an equation $A(x)=b$ have the form $A(x)=b+R$ with a small perturbation $R$. An approximate solution $X$ of $A(x)=b$ with residual $R(X)=A(X)-b$, thus can be viewed as an exact solution of the perturbed equation $A(X)=b+R \equiv \bar{b}$, with here an $x$ satisfying $A(x)=b$ appearing as an approximate solution satisfying $A(x)=\bar{b}-R$. For a wellposed solution the distinction between an exact solution $x$ and and approximate solution $X$ disappears, since both can be viewed as equally representative solutions to perturbed equations.

Wellposedness of a certain output functional $M(x)$ of the solution $x$ to an equation $A(x)=b$ with respect to perturbations of data $b$, can be expressed in a stability estimate of the form

$$
|M(x)-M(X)| \leq S\|R\|,
$$

where $A(X)=b+R, S$ is a stability factor, and $\|\cdot\|$ is a certain residual norm. The output $M(x)$ is wellposed with respect to residual perturbations measured in the $\|\cdot\|$-norm, if $S$ is of moderate size in the sense that $S\|R\|$ can be made small. In a wellposed problem, small residual perturbations thus have small effects on the output.

Note that sometimes wellposedness is replaced by "continuous dependence on data" without the quantitative requirement that the dependence should be Lipschitz continuous with Lipschitz constant of moderate size, which is needed to express that small perturbations have small output effects. However, without this quantitative aspect, the distinction between wellposed and illposed problem becomes blurred and both scientific and mathematical rigor suffers. This criticism extendss to the use of smooth or $C^{\infty}$ without quantitative measure of the size of derivatives, as in the formulation of the Millennium problem [7].

\section{The Role of Computation}

The above discussion is motivated by a common attitude among both pure and applied mathematicians that computed solutions to differential equations "prove nothing". This belief is probably rooted in the classical a priori error analysis 
of difference methods for computing solutions to differential equations, based on the concept of truncation error, which is the residual obtained inserting the exact solution into the difference equation of the difference method. However, this corresponds to a thought experiment, since the exact solution is unknown along with the truncation error. Nevertheless the exact solution is given the major role and is viewed as an approximate solution of the difference equation, while the finite difference solution is not viewed as an approximate solution of the differential equation.

In the a posteriori error analysis of finite element methods including EG2, instead the computed solution is inserted into the differential equation resulting in a computable residual. The computed finite element solution thus has the major role and in case of wellposedness is a representive solution as much as any exact solution subject to perturbations. This is a key aspect with potentially farreaching consequences as concerns the role of computation in the mathematics of solving wellposed (differential) equations.

In the case of the Euler equations, we shall see wellposed EG2-solutions taking over the whole scene, since wellposed exact global solutions simply do not seem to exist. The thought experiment of inserting a wellposed exact solution into an Euler difference equation, thus may have no meaning, while inserting a wellposed computed finite element solution into the Euler equations definitely has a meaning.

\section{Blowup for Compressible Flow}

The Euler equations express conservation of mass, momentum and total energy of a fluid with vanishingly small viscosity (inviscid fluid). In the case of compressible flow, it is well known that initially smooth solutions to the Euler equations in general develop into discontinuous shock solutions in finite time. Such shock solutions thus exhibit blowup in the sense that they develop infinitely large derivatives and Euler residuals at the shock and there violate the Euler equations pointwise. The formation of shocks shows non-existence of pointwise solutions to the compressible Euler equations. Concepts of weak solution have been developed accomodating after-blowup shock solutions with Euler residuals being large in a strong (pointwise) sense and vanishingly small in a weak sense (combined with entropy inequalities).

Viscosity solutions of the Euler equations are solutions to regularized Euler equations augmented by viscous terms with small viscosity coefficients. Proving convergence of viscosity solutions to weak solutions of the Euler equations as the viscosity tends to zero, is a longstanding open problem, only settled in very restricted model cases. Even the problem of existence of viscosity solutions is open in the general case [8].

A way out of this unfortunate stalemate is offered by computation: EG2solutions can be viewed as specific viscosity solutions of the Euler equations with specific viscous regularization depending on the mesh size, and since EG2 solutions are computed they exist and are available for inspection. This is analogous to the existence proof by Descartes based on the a posteriori observation: Cog- 
ito, ergo sum or I think, therefore I exist, (as compared to an analytical/logical a priori proof of the existence of God attempted by many philosophers without clear success). A computational foundation of thermodynamics based on EG2 for compressible flow is developed on [14].

\section{The Blowup Problem for Incompressible Flow}

Incompressible flow does not form shocks and one may ask if initially smooth solutions of the incompressible Euler equations exhibit blowup or not, which is the Millennium Problem in the case of vanishing viscosity. The existing literature, see $[5,10,11,12]$ and references therein, is not decisive and evidence for both blowup and not blowup is presented. The study has further been limited in time to before blowup, discarding the highly relevant question of what happens after blowup.

In this note we present evidence of blowup for the incompressible Euler equations drawing from our recent work [13] and references therein, widening the study to both before and after blowup. We compute specific viscosity solutions by EG2 and we discover wellposedness of mean-value outputs such as drag and lift (coefficients), showing that EG2 solutions are representative Euler solutions. We find that the phenomenon of turbulence in incompressible flow, plays a similar role in blowup as that of shock formation in compressible flow: Initially smooth viscosity solutions of the incompressible Euler equations in general in finite time show blowup into turbulent solutions, characterized by pointwise large (weakly small) Euler residuals and substantial turbulent dissipation. We give evidence that the blowup into turbulence results from pointwise instability, forcing smooth solutions to develop into turbulent solutions, as a parallel to the inevitable shock formation in compressible flow. Since viscosity solutions are turbulent with derivatives becoming unbounded in turbulent regions, a limit would there be nowhere differentiable and have infinitely small scales, like a very complex Weierstrass function. No evidence for the existence of such a limit seems to be available; only viscosity solutions are thinkable and computed EG2 solutions do exist.

In the work cited in $[5,6,10,11,12]$, blowup is identified by the development of an infinite velocity gradient of a specific initially smooth exact Euler solution as time approaches a blowup time. This form of blowup detection, which we refer to as local blowup, requires pointwise accurate information (analytically or computationally) of the blowup to infinity, which (so far) has shown to be impossible to obtain. In this approach, wellposedness is left out assuming no perturbations from data or solution procedure. Further, the question of what happens beyond blowup is left out. Altogether, the approach of local blowup has not been conclusive.

We use a different approach based on convergence of globally defined EG2 solutions under decreasing mesh-size, taking wellposedness into account. Blowup is then identified by increasing space-time $L_{2}$-norms of Euler residuals with decreasing mesh size. We give evidence that blowup can be detected by computation with finite mesh size thus without requiring the mesh size to tend to zero, 
which is impossible to realize. We also include the process after blowup. In this approach, which we refer to as global blowup, we thus avoid the seemingly impossible task of a providing a precise analysis of the route to local blowup of a smooth exact Euler solution. Instead we observe initial smooth potential flow develop into turbulent flow identified by increasing $L_{2}$-residuals under decreasing mesh size. In this approach there is no pointwise unique route to blowup with a unique blowup time, since the transition to turbulence feeds on the mesh-dependent residual perturbations in EG2 computation. We show that the transition to turbulence in potential flow is driven by exponential perturbation growth in time with corresponding logarithmic growth in the mesh size of the effective time to transition. We thus study global blowup of EG2/viscosity solutions under decreasing mesh size/viscosity including wellposedness, and not as in $[5,10,11,12]$ local blowup of exact Euler solutions without wellposedness.

Fefferman's problem formulation does not give a precise definition of blowup, other than the converse of global existence of a smooth solution, with a vague definition of "smooth". Again we use his "lee-way" and suggest to define blowup in terms of global blowup of viscosity solutions as indicated. We note that the approach of local blowup is taken over from the basic existence theory of ordinary differential equations, where divergence to infinity in time is the only way existence can be violated. However, partial differential equations such as the Euler equations have a much richer blowup structure in space (when subject to perturbations), which seems to be inaccessible to a pointwise analysis.

\section{Blowup Detection by Finite Mesh Size Computation}

A key issue in global blowup detection by computation is if a finite mesh size computation can be sufficient. Let us first address this question in an elementary example: Consider the non-smooth shock solution $u(x)=1$ for $x<0$ and $u(x)=-1$ for $x>0$ of the stationary Burgers equation $u u^{\prime}=0$, where $u^{\prime}=\frac{\partial u}{\partial x}$, which is a $1 \mathrm{~d}$ model of the (compressible) Euler equations. This is a typical Burgers solution, which is a piecewise smooth function with a finite number of jump discontinuities. Let $h>0$ and define $U_{h}(x)=1$ for $x<-h, U_{h}(x)=-\frac{x}{h}$ for $-h \leq x \leq h, U_{h}(x)=-1$ for $x>h$, as a corresponding viscosity solution. We have

$$
\int_{-h}^{h}\left(U_{h} U_{h}^{\prime}\right)^{2} d x=\frac{2}{3 h}
$$

and thus the $L_{2}$-norm of the Burgers residual $U_{h} U_{h}^{\prime}$ scales like $h^{-1 / 2}$. Detecting $h^{-1 / 2}$ increase of $L_{2}$-residuals under decreasing mesh size would then be identified with blowup into a shock, since in smooth parts the residual would decrease like $h$. In this case the shock would be correctly detected with a finite mesh size.

We may compare with detecting shocks by local blowup, resolving the flow pointwise as the shock is forming. This requires mesh refinement without limit, and like Achilles will never reach the goal.

More generally, to accurately detect a shock in a Burgers solution $u$, the smooth part of $u$ has to be resolved in order to correctly single out a shock from a smooth part. Now, the general structure of Burgers solutions as being 
piecewise smooth with jumps allows detection with a finite mesh size. The structure of Burgers solutions with a resolvable smooth part and sharp shocks with no smallest scale, thus makes blowup detection possible without requiring the mesh size to be infinitely small. Thus beyond the resolvable scale of the smooth part there can be no surprises to be found by decreasing the mesh size, since all there is are shocks separated by smooth parts.

Massive evidence indicates that incompressible Euler solutions have a similar general structure with a smooth (laminar) part resolvable with finite mesh size combined with a turbulent part with no smallest scale and thus unresolvable on all meshes. Again we have a dichotomy into a resolvable and an unresolvable part, allowing accurate blowup detection with finite mesh size/viscosity. Massive evidence indicates that a flow becomes partly turbulent if the Reynolds number $R e$ is large enough, where $R e=\frac{U L}{\nu}$ with $U$ a representative velocity, $L$ a length scale and $\nu$ the viscosity. In particular, there is massive evidence that if a flow is partly turbulent for a particular Reynolds number, it will remain so under decreasing viscosity and increasing Reynolds number. This can be motivated by rescaling decreasing the length scale with the viscosity (thus focussing on a part of the fluid domain) keeping the Reynolds number constant and thus expecting the flow to remain turbulent.

This opens the possibility of detecting blowup of Euler solutions from finite mesh size computation, in particular because EG2 solutions satisfy slip boundary conditions and thus lack the thin boundary layers of slightly viscous NavierStokes solutions satisfying no-slip boundary conditions, which are unresolvable on any foreseeable computer. EG2 thus introduces viscous regularization in the interior of the fluid domain combined with a weak Neumann velocity condition in the tangential direction allowing slip. This is motivated by the experimentally observed fact that the skin friction from non-zero tangential velocities on the boundary, tends to zero as the viscosity tends to zero. This connects back to Eulers original idea of the Euler equations with slip as a useful model of slightly viscous flow, as developed in detail in [13], in contrast to the legacy of Prandtl discarding Euler solutions because they do not satisfy no-slip boundary conditions, thus requiring the resolution of thin boundary layers of Navier-Stokes solutions, which however is impossible. We find that EG2/slip allows simulation of flows with large Reynolds numbers $\left(R e \geq 10^{6}\right.$ say) on meshes with $10^{6}$ mesh points, rather than $10^{16}$ as required for no-slip according to state-of-the-art CFD [16].

The Euler equations with slip boundary conditions thus are useful, because solutions can be computed by EG2 and can provide important information, such as the drag of a car, see Fig. 20.3 below, while the Navier-Stokes equations with non-slip boundary conditions seem less useful, because solutions cannot be computed. In particular, there may be strong reason to include the Euler equations as a most useful limit case of the Navier-Stokes Millenium Problem, instead of discarding it on formal reasons. 


\section{Evidence of Blowup of Incompressible Flow}

For the discussion we focus on blowup of potential flow around a circular cylinder, and refer to a variety of similar results in our cited work. We summarize the evidence in [13] for blowup of Euler solutions starting from smooth potential flow as follows:

(a) EG2 solutions are representative viscosity solutions to the Euler equations in the sense that mean value outputs such as drag and lift coefficients and total turbulent dissipation, are wellposed under $H^{-1}$ residual perturbations.

(b) EG2 solutions show under finite mesh refinement substantial turbulent dissipation with $L_{2}$-residuals increasing like $h^{-1 / 2}$, while $H^{-1}$-residuals decrease like $h^{1 / 2}$.

(c) Outputs such as drag and lift of the potential solution have exponentially large computed stability factors.

(d) The potential solution is linearly pointwise exponentially unstable.

(e) Detecting blowup into turbulence in an EG2 computation with a certain finite mesh size, indicates blowup also for smaller mesh sizes.

Here (a)-(c) are shown by computation, while (d) follows by a simple analytical argument. Now, (c) supported by (d) shows that the potential solution is not wellposed and thus is not a representative solution. On the other hand, by (a) EG2 solutions are wellposed representative solutions and since they by (b) show to be turbulent, they give computational evidence of blowup of the potential solution. Since the potential solution is resolvable with finite mesh size, there is strong evidence that the turbulent blowup detection is correct.

Note that (e) is motivated by the fact that the effective Reynolds number in EG2 scales with $h^{-1}$, so that decreasing the mesh size increases the effective Reynolds number, and thus by the above scaling argument turbulence cannot disappear under mesh refinement. Of course a pure mathematician could question this statement, claiming that we have not "proved" that decreasing the mesh size/viscosity could not indicate instead non blowup. Of course, the question is what exactly is meant by a "proof". Ultimately it must come down to an agreement among specialists. Of course, one may turn the question around and ask anyone disputing the correctness of the blowup detection, to present any argument supporting the idea that further decrease of mesh size/viscosity could turn a turbulent solution into a fully laminar solution. We believe there is no such argument.

We may compare with Perleman's proof of the Poincaré conjecture, another Millennium Problem, which is now under scrutiny by a group of specialists filling in many missing details of Perelman's original argument. Will it be possible to work out every detail into a formal proof beyond any possible question by anybody? If so, why did not Perelman write down this proof to start with? 
The wellposedness (a) follows from observing that the stability factor $S$ in the form of the $H^{1}$-norm of the solution to a dual linearized problem, linearized at computed solutions, is of moderate size. An EG2 solution with residual $R$ and stability factor $S$ is thus showed to be representative by showing that it passes the test

$$
S R \leq T O L
$$

In order to be a representative solution, it is not enough to have a small residual $R$; in addition $S R$ has to be small according to (8.1). This test will disqualifies the potential solution as a representative solution because the corresponding $S$ is exponentially large. The stability test (8.1) is a new crucial tool offered by computation, inaccessible to mere analysis.

We sum up: EG2 solutions are specific viscosity solutions of the Euler equations with specific viscosity arising from least squares penalization of the Euler residual, roughly speaking corresponding to introducing a standard viscosity proportional to the mesh size. Inspecting EG2 solutions we find that they are turbulent, in the sense that their Euler residuals are small weakly but large strongly, and wellposed in the sense that certain mean-value outputs are stable under mesh refinement. In short, we present evidence that the Euler equations have wellposed non-smooth turbulent solutions for a variety of data, but no smooth wellposed solutions. We detect blowup into turbulence with finite mesh size and use a scaling argument to take this as evidence of blowup for smaller mesh size in accessible to computation. We thus argue that blowup can be detected by finite mesh size computation.

The computations in [13] were performed on a PC on meshes with up to 200.000 mesh points. We are now computing consistent results on a Blue Gene with 2000 processors on meshes with up to 100 million mesh points. These new results will be published shortly.

\section{The Tolerance}

For turbulent solutions there is for each choice of output a minimal possible tolerance $T O L_{m}>0$. For example, if the output is the instantaneous drag $d(t)$ at time $t$, then $T O L_{m}$ cannot be smaller than the amplitude $\delta$ of the fluctuation of $d(t)$ in time, and $\delta>0$. If the output is a drag coefficient, that is a certain meanvalue in time of $d(t)$, that is a meanvalue over a certain number $M$ of periods of fluctuation of $d(t)$, then it may be expected that

$$
T O L_{m} \approx \frac{\delta}{M^{\theta}}
$$

where $0.5 \leq \theta \leq 1$. In other words, the output of turbulent flow is not determined up to a certain minimal tolerance, and once this tolerance has been reached in computation, further mesh refinement does not improve the quality of the output information. In practice, one may have with suitable normalization $T O L_{m} \approx 0.1$ for $d(t), T O L_{m} \approx 0.01$ for drag coefficients, but rarely $T O L_{m} \approx 0.001$ for any output. 
This is different from laminar flow, for which with sufficient mesh refinement any tolerance can be reached even for pointwise outputs. For turbulent flow the minimal tolerance thus can be reached with a finite mesh size, while for laminar flow further mesh refinement always decreases the tolerance.

EG2 finite mesh size computation for turbulent flow, thus can give best possible output information, and a computed finite mesh size EG2 solution thus can be fully representative in the sense that there is no better representative. This supports the claim of this note that blowup of Euler solutions can be proved by EG2 finite mesh size computation. The issue thus is not to compute on finest possible meshes using maximal computational power, but rather the opposite.

\section{Smooth Solutions and Non-Blowup}

In $[2,4]$ a technique related to $(2.1)$ is presented for proving existence of a strong solution to the Navier-Stokes equations by computation: It is proved that (i) if an approximate solution has a sufficiently small residual, then a strong (smooth) exact solution exits, and (ii) if a smooth exact solution exists, then there is an approximate solution with strongly small residual. The net result of [4] is thus that existence of a smooth exact solution, and thus non-blowup, can be verified by computation, with the important qualification of sufficiently fine mesh size in order to guarantee sufficiently small residual. In other words the implication $\operatorname{not} B \rightarrow \operatorname{not} A$ is proved in [4], where $A=$ blowup of exact solution, and $B=$ blowup of Galerkin approximate solutions. The trouble with this implication is that not $B$ is never true, not even under vanishing mesh size: Computed solutions with finite mesh size show blowup which does not disappear under vanishing mesh size. The implication $\operatorname{not} B \rightarrow \operatorname{not} A$ thus is empty.

On the other hand, we give evidence that blowup can correctly be detected by computations with finite mesh size based on the observed fact that full output information of turbulent Euler solutions can be obtained by finite mesh size computation. This is in contrast to computation of smooth solutions without blowup for which no finite mesh size can give full information. To sum up, turbulent flow is ideally suited to computation, since analytical information is fully missing and computation can give full information.

\section{The Incompressible Euler Equations}

We recall the Euler equations expressing conservation of momentum and mass of an incompressible inviscid fluid enclosed in a volume $\Omega$ in $\mathbb{R}^{3}$ with boundary $\Gamma$ : Find the velocity $u=\left(u_{1}, u_{2}, u_{3}\right)$ and pressure $p$ depending on $(x, t) \in \bar{\Omega} \times I$ with $\bar{\Omega}=\Omega \cup \Gamma$, such that

$$
\begin{aligned}
\dot{u}+(u \cdot \nabla) u+\nabla p & =f & & \text { in } \Omega \times I, \\
\nabla \cdot u & =0 & & \text { in } \Omega \times I, \\
u \cdot n & =g & & \text { on } \Gamma \times I, \\
u(\cdot, 0) & =u^{0} & & \text { in } \Omega,
\end{aligned}
$$

where $n$ denotes the outward unit normal to $\Gamma, f$ is a given volume force, $g$ is a given inflow/outflow velocity, $u^{0}$ is a given initial condition, $\dot{u}=\frac{\partial u}{\partial t}$ and 
$I=[0, T]$ a given time interval. We notice the slip boundary condition expressing inflow/outflow with zero friction.

The Euler equations in the pointwise form (11.1) look deceptively simple, but have a major drawback: They cannot be solved exactly in a pointwise (not even weak) sense, because of blowup by exponential instability into turbulence!

\section{Exponential Instability}

The lack of viscosity with regularizing effect make the Euler equations inaccessible to an analytical mathematical study. The difficulty is exposed by formal linearization: Subtracting the Euler equations for two solutions $(u, p)$ and $(\bar{u}, \bar{p})$ with corresponding (slightly) different data, we obtain the following linearized equation for the difference $(v, q) \equiv(u-\bar{u}, p-\bar{p})$ :

$$
\begin{aligned}
\dot{v}+(u \cdot \nabla) v+(v \cdot \nabla) \bar{u}+\nabla q & =f-\bar{f} & & \text { in } \Omega \times I, \\
\nabla \cdot v & =0 & & \text { in } \Omega \times I, \\
v \cdot n & =g-\bar{g} & & \text { on } \Gamma \times I, \\
v(\cdot, 0) & =u^{0}-\bar{u}^{0} & & \text { in } \Omega .
\end{aligned}
$$

Formally, with $u$ and $\bar{u}$ given, this is a linear convection-reaction problem for $(v, q)$ with the reaction term given by the $3 \times 3$ matrix $\nabla \bar{u}$. By the incompressiblity, the trace of $\nabla \bar{u}$ is zero, which shows that in general $\nabla \bar{u}$ has eigenvalues with real value of both signs, of the size of $|\nabla u|$ (with $|\cdot|$ some matrix norm), thus with at least one exponentially unstable eigenvalue. Thus we expect to generically find exponential perturbation growth with exponent $|\nabla u|$. In particular, as we will see below, a smooth stationary potential solution is unstable and under infinitesimal perturbations in finite time develops into a turbulent solution with $|\nabla u|$ large, as an analogue to the formation of shocks in compressible flow. Birkhoff questions in [3] if there is any stable Euler solution, but gets a devastating review in [18]. Fefferman states in [7]: "Many numerical computations appear to exhibit blowup for solutions of the Euler equations, but the extreme numerical instability of the equations makes it very hard to draw reliable conclusions". It is natural to ask why Fefferman views "the extreme numerical instability of the equations", not as a sign of instability and blowup, but only as an obstacle to conclusion.

\section{Gronwall Stability Estimates}

In the analytical mathematical theory of partial differential equations, Gronwall type estimates are commonly used to assess the stability of the linearized problem (12.1). A typical such estimate is obtained multiplying the momentum equation of (12.1) by $v$, assuming $\bar{f}=f$ and $\bar{g}=g$, and integrating in space to obtain an estimate of the form

$$
\frac{d w}{d t} \leq\|\nabla \bar{u}\|_{\infty} w \quad \text { on } I
$$


where $w(t)=\|v(t)\|_{0}^{2}$ with $\|\cdot\|_{0}$ the $L_{2}(\Omega)$ norm and $\|\cdot\|_{\infty}$ the $L_{\infty}(\Omega)$-norm. By integration in time it follows that

$$
w(T) \leq \exp (C) w(0)
$$

where

$$
C=C(\nabla \bar{u})=\exp \left(\int_{0}^{T}\|\nabla \bar{u}(t)\|_{\infty} d t\right) .
$$

It is then commonly argued that if $C<\infty$, then also $\exp (C)<\infty$, and in particular that $w(T)=0$ if $w(0)=0$ showing uniqueness.

However, insisting that $C<\infty$ and $\exp (C)<\infty$ express the same mathematical inequality does not (as in the Clay problem formulation) acknowledge the importance of Hadamard's distinction between a wellposed problem with moderate stability factor $(C)$ and an illposed problem with large stability factor $(\exp (C))$. Already the very moderate values $\|\nabla \bar{u}\|_{\infty}=20$ and $T=15$ will give $\exp (C)=\exp (300) \sim 10^{100}=$ googol which is much bigger than the total number of atoms on the Universe estimated to be around $10^{80}$. A solution with stability factor of googol size is not wellposed. Note that Google derives its name from googol; making no distinction between $10^{100}$ and 100 would be like making no distinction beween the entire Google and one single paragraph, or more precisely making no distintion between a natural number $n$ and the totality of numbers formed by $n$ digits.

For potential flow, $|\nabla \bar{u}| \sim 1$ indicating a perturbation growth of $\exp (T)$ and thus a transition time $T \sim \log (1 / h)$, since the residual perturbation is of size $h$ as long as the solution is smooth. We thus expect to see a weak logarithmic dependence of the transition time on the mesh size (since all perturbations come from the EG2 mesh computation).

We emphasize the importance of making a distinction between $C$ and $\exp (C)$ in connection with wellposedness. Without this distinction Hadamard's fundamental concept of wellposedness looses its meaning along with mathematical analysis of differential equations.

\section{The Vorticity Equation}

Formally applying the curl operator $\nabla \times$ to the momentum equation we obtain the vorticity equation

$$
\dot{\omega}+(u \cdot \nabla) \omega-(\omega \cdot \nabla) u=\nabla \times f \quad \text { in } \Omega,
$$

which is a convection-reaction equation in the vorticity $\omega=\nabla \times u$ with coefficients depending on $u$, of the same form as the linearized equation (12.1), with similar properties of exponential instability referred to as vortex stretching. The vorticity is thus locally subject to exponential growth with exponent $|\nabla u|$.

In classical analysis it is often argued that from the vorticity equation (20.2), it follows that vorticity cannot be generated starting from potential flow with zero vorticity and $f=0$, which is Kelvin's theorem. But this is an incorrect conclusion, since perturbations of $\bar{f}$ of $f$ with $\nabla \times \bar{f} \neq 0$ must be taken into 
account. What you effectively see in computations is local exponential growth of vorticity in vortex stretching, even if $\nabla \times f=0$, which is a main route of instability to turbulence.

\section{The Beale-Kato-Majda Blowup Criterion}

A corner stone in the approach of local blowup presented in $[5,6]$ is a result from 1984 by Beale-Kato-Majda [1] stating that "the maximum norm of the vorticity alone controls the breakdown of smooth solutions for the $3 \mathrm{~d}$ Euler equations": An initially smooth Euler solution does not blow up on $[0, T]$ if and only if

$$
C(\omega)=\int_{0}^{T}\|\omega(t)\|_{\infty} d t<\infty,
$$

(or replacing $\omega$ with the symmetric part of $\nabla u[17]$ ). This result is based on Gronwall estimates without distinction between $C(\omega)$ and $\exp (C(\omega))$ and without taking illposedness with exponential perturbation growth of size $\exp (C(\omega))$ into account, and thus its relevance can be questioned.

\section{Viscous Regularization}

We define the Euler residual by

$$
R(u, p) \equiv f-(\dot{u}+(u \cdot \nabla) u+\nabla p),
$$

which is the residual of the momentum equation, assuming for simplicity that the incompressiblity equation $\nabla \cdot u=0$ is not subject to perturbations. The regularized Euler equations take the form: Find $\left(u_{\nu}, p_{\nu}\right)$ such that

$$
\begin{aligned}
R\left(u_{\nu}, p_{\nu}\right) & =-\nabla \cdot\left(\nu \nabla u_{\nu}\right) & & \text { in } \Omega \times I, \\
\nabla \cdot u_{\nu} & =0 & & \text { in } \Omega \times I, \\
u_{\nu} \cdot n & =g & & \text { on } \Gamma \times I, \\
u_{\nu}(\cdot, 0) & =u^{0} & & \text { in } \Omega,
\end{aligned}
$$

where $\nu>0$ is a small viscosity, together with a homogeneous Neumann boundary condition for the tangential velocity. Notice that we keep the slip boundary condition $u_{\nu} \cdot n=g$, which eliminates viscous Dirichlet no-slip boundary layers. The turbulence we will discover thus does not emanate from viscous no-slip boundary layers (which is a common misconception). We consider here for simplicity a standard ad hoc regularization and return to computational regularization below. Existence of a pointwise solution $\left(u_{\nu}, p_{\nu}\right)$ of $(16.2)$ (allowing $\nu$ to have a certain dependence on $|\nabla u|)$, follows by standard techniques, see e.g. [5]. Notice that the Euler residual $R\left(u_{\nu}, p_{\nu}\right)$ equals the viscous term $-\nabla \cdot\left(\nu \nabla u_{\nu}\right)$, which suggests an interpretation of the viscous term in the form of the Euler residual.

The standard energy estimate for (16.2) is obtained by multiplying the momentum equation with $u_{\nu}$ and integrating in space and time, to get in the case 
$f=0$ and $g=0$,

$$
\int_{0}^{t} \int_{\Omega} R\left(u_{\nu}, p_{\nu}\right) \cdot u_{\nu} d x d t=D\left(u_{\nu} ; t\right) \equiv \int_{0}^{t} \int_{\Omega} \nu\left|\nabla u_{\nu}(s, x)\right|^{2} d x d s
$$

from which follows by standard manipulations of the left hand side,

$$
K\left(u_{\nu}(t)\right)+D\left(u_{\nu} ; t\right)=K\left(u^{0}\right), \quad t>0,
$$

where

$$
K\left(u_{\nu}(t)\right)=\frac{1}{2} \int_{\Omega}\left|u_{\nu}(t, x)\right|^{2} d x .
$$

This estimate shows a balance of the kinetic energy $K\left(u_{\nu}(t)\right)$ and the viscous dissipation $D\left(u_{\nu} ; t\right)$, with any loss in kinetic energy appearing as viscous dissipation, and vice versa. In particular $D\left(u_{\nu} ; t\right) \leq K(0)$ and thus the viscous dissipation is bounded (if $f=0$ and $g=0$ ).

Turbulent solutions of (16.2) are characterized by substantial turbulent dissipation, that is (for $t$ bounded away from zero),

$$
D(t) \equiv \lim _{\nu \rightarrow 0} D\left(u_{\nu} ; t\right)>>0 .
$$

That a substantial positive limit exists is Kolmogorov's conjecture, which is consistent with

$$
\left\|\nabla u_{\nu}\right\|_{0} \sim \frac{1}{\sqrt{\nu}}, \quad\left\|R\left(u_{\nu}, p_{\nu}\right)\right\|_{0} \sim \frac{1}{\sqrt{\nu}},
$$

where $\|\cdot\|_{0}$ denotes the $L_{2}(Q)$-norm with $Q=\Omega \times I$. On the other hand, it follows by standard arguments from (16.4) that

$$
\left\|R\left(u_{\nu}, p_{\nu}\right)\right\|_{-1} \leq \sqrt{\nu}
$$

where $\|\cdot\|_{-1}$ is the norm in $L_{2}\left(I ; H^{-1}(\Omega)\right)$. Kolmogorov thus conjectures that the Euler residual $R\left(u_{\nu}, p_{\nu}\right)$ is strongly (in $L_{2}$ ) large, while being small weakly (in $H^{-1}$ ).

\section{EG2 Regularization}

An EG2 solution $(U, P)$ on a mesh with local mesh size $h(x, t)$ according to [13], satisfies the following energy estimate (with $f=0$ and $g=0$ ):

$$
K(U(t))+D_{h}(U ; t)=K\left(u^{0}\right)
$$

where

$$
D_{h}(U ; t)=\int_{0}^{t} \int_{\Omega} h R(U, P)^{2} d x d t,
$$

is an analog of $D\left(u_{\nu} ; t\right)$ with $h \sim \nu$. We see that the EG2 viscosity arises from penalization of a non-zero Euler residual $R(U, P)$ with the penalty directly connecting to the violation (according the theory of criminology). A turbulent 
solution is characterized by substantial dissipation $D_{h}(U ; t)$ with $\|R(U, P)\|_{0} \sim$ $h^{-1 / 2}$, and

$$
\|R(U, P)\|_{-1} \leq \sqrt{h}
$$

in accordance with (16.6) and (16.7). Inspection of computed EG2 solutions show that they are turbulent with substantial turbulent dissipation.

EG2 explains the occurence of viscous effects in Euler solutions in a new way, not simply assuming ad hoc that "there is always some small constant shear viscosity", but from the impossibility of pointwise exact conservation of momentum. EG2 viscosity is not a simple constant shear viscosity but rather a solution dependent bulk (or streamline) viscosity $[13,14]$.

\section{Wellposedness of Mean-Value Outputs}

Let $M(v)=\int_{Q} v \psi d x d t$ be a mean-value output of a velocity $v$ defined by a smooth weight-function $\psi(x, t)$, and let $(u, p)$ and $(U, P)$ be two EG2-solutions on two meshes with maximal mesh size $h$. Let $(\varphi, \theta)$ be the solution to the dual linearized problem

$$
\begin{aligned}
-\dot{\varphi}-(u \cdot \nabla) \varphi+\nabla U^{\top} \varphi+\nabla \theta & =\psi & & \text { in } \Omega \times I, \\
\nabla \cdot \varphi & =0 & & \text { in } \Omega \times I, \\
\varphi \cdot n & =g & & \text { on } \Gamma \times I, \\
\varphi(\cdot, T) & =0 & & \text { in } \Omega,
\end{aligned}
$$

where $\top$ denotes transpose. Multiplying the first equation by $u-U$ and integrating by parts, we obtain the following output error representation $[13,14]$

$$
M(u)-M(U)=\int_{Q}(R(u, p)-R(U, P)) \cdot \varphi d x d t
$$

from which follows the a posteriori error estimate as an analog to (3.1):

$$
|M(u)-M(U)| \leq S\left(\|R(u, p)\|_{-1}+\|R(U, P)\|_{-1}\right),
$$

where the stability factor

$$
S=S(u, U, M)=S(u, U)=\|\varphi\|_{H^{1}(Q)} .
$$

In [13] we present a variety of evidence, obtained by computational solution of the dual problem, that for global mean-value outputs such as drag and lift, $S<<$ $1 / \sqrt{h}$, while $\|R\|_{-1} \sim \sqrt{h}$ in conformity with (16.7), allowing EG2 solutions to pass the wellposedness test (8.1) for $T O L \geq T O L_{m}$. The minimal tolerance $T O L_{m}$ is determined computationally by observing stationarity of output quality under mesh refinement [13]. We thus find evidence of wellposedness of meanvalue outputs of EG2 solutions to tolerances of interest showing that finite mesh size EG2 solutions are representative solutions of the Euler equations.

Similarly we may test the wellposedness of any approximate Euler solution $(u, p)$ by the corresponding stability factor $S(u, u)$. Given two approximate 
solutions $(u, p)$ and $(U, P)$, there are thus four related stability factors, namely $S(u, u), S(u, U), S(U, u)$ and $S(U, U)$ connecting to four dual problems with various (perturbations) of the advection/reaction coefficients. We give in [13] evidence that for EG2 solutions, these stability factors are of the same moderate size. If $(u, p)$ is an unstable potential solution, then $S(u, u)$ is very large which disqualifies a potential solution as a non-wellposed solution without significance.

We can thus test wellposedness of a single solution $(u, p)$ on a single mesh, typically and EG2 solution, by the size of the corresponding stability factor $S(u, u)$. This is analogous to testing the wellposedness of a solution $x$ to a scalar equation $A(x)=b$ by the size of the stability factor $S=\left|A^{\prime}(x)^{-1}\right|$. For a single solution with moderate size $S(u, u)$, the output effect of a (sufficiently small) residual perturbation $d R$ is bounded by $S(u, u)\|d R\|_{-1}$. For two EG2 solutions $(u, p)$ and $(U, P)$ the stability factors $S(u, U)$ and $S(U, u)$ offer tests of wellposedness for residual perturbations which are not necessarily very small.

\section{Stability of the Dual Linearized Problem}

A crude analytical stability analysis of the dual linearized problem (18.1) using Gronwall type estimates, indicates that the dual problem is pointwise exponentially unstable because the reaction coefficient $\nabla U$ is locally very large, in which case effectively $S=\infty$. This is consistent with massive observation that pointvalues of turbulent flow are non-unique or unstable.

On the other hand we observe computationally that $S$ is not large for meanvalue outputs of turbulent solutions. We explain in [13] this remarkable fact as an effect of cancellation from the following two sources:

(i) rapidly oscillating reaction coefficients of turbulent solutions,

(ii) smooth data in the dual problem for mean-value outputs.

For a laminar solution there is no cancellation, and therefore not even meanvalues are unique. This is d'Alembert's paradox: A potential laminar solution has zero drag, while an arbitrarily small perturbation will turn it into a turbulent solution with substantial drag. The drag of a laminar solution is thus nonunique in the sense that an arbitrarily small perturbation will change the drag substantially. The stability factor is infinite for a laminar solution because of lack of cancellation [13].

\section{Flow around Circular Cylinder}

We now present one of several examples of blowup in [13]. We consider potential flow (stationary inviscid incompressible irrotational flow) of a fluid of unit density filling $\mathbb{R}^{3}$ with coordinates $x=\left(x_{1}, x_{2}, x_{3}\right)$ and moving with velocity $(1,0,0)$ at infinity, around a circular cylinder of unit radius with axis along the $x_{3}$-axis. We recall that the potential flow is constant in the $x_{3}$-direction and is given (in polar coordinates $(r, \theta)$ in a $\left(x_{1}, x_{2}\right)$-plane) by the potential function

$$
\varphi(r, \theta)=\left(r+\frac{1}{r}\right) \cos (\theta)
$$


with corresponding velocity components

$$
u_{r} \equiv \frac{\partial \varphi}{\partial r}=\left(1-\frac{1}{r^{2}}\right) \cos (\theta), \quad u_{s} \equiv \frac{\partial u}{\partial s} \equiv \frac{1}{r} \frac{\partial \varphi}{\partial \theta}=-\left(1+\frac{1}{r^{2}}\right) \sin (\theta) .
$$

This is a fully symmetric solution with zero drag, see Fig. 20.1.

We find that an EG2 solution initiated as the potential flow with zero drag over time exhibits blowup into a turbulent solution with substantial turbulent dissipation in a low pressure turbulent wake, as displayed in Fig. 20.1, with substantial drag with drag coeffient $\approx 1.0$ in accordance with experiments for high Reynolds number flow (beyond the drag crisis). In particular we find strong vorticity in the streamline direction attaching at rear separation coupled with low pressure generating drag. Below we describe a scenario based on the linearized equations for the blowup of the potential solution into the turbulent solution with strong streamwise vorticity generated at rear separation.

We observe [13] a (relative) fluctuation of the momentary drag $d(t)$ of about $20 \%$ with corresponding minimal (relative) tolerance $T O L_{m} \approx 0.2$. For the dragcoefficient, which is a mean-values in time, we may reach $T O L_{m} \approx 0.01$ according to aposteriori estimation by duality [13], while a relative tolerance of 0.001 may be uattainable. We thus find that the drag of EG2 solutions is wellposed to a certain minimal tolerance $T O L_{m} \approx 0.01$. We thus find EG2 solutions to be representative solutions with blowup and we thus obtain evidence of (II).

\section{An Analysis of Blowup at Rear Separation}

We now analyze the mechanism underlying the generation of low pressure streamwise streaks at rear separation through the linearized problems (12.1) in velocity-pressure and (20.2) in vorticity. As a model of potential flow at rear separation, we consider the potential flow $u=\left(x_{1},-x_{2}, 0\right)$ in the half-plane $\left\{x_{1}>0\right\}$. Assuming $x_{1}$ and $x_{2}$ are small, we approximate the $\varphi_{2}$-equation of (12.1) by

$$
\dot{\varphi}_{2}-\varphi_{2}=f_{2}
$$

where $f_{2}=f_{2}\left(x_{3}\right)$ is an oscillating mesh residual perturbation depending on $x_{3}$ (including also a pressure-gradient), for example $f_{2}\left(x_{3}\right)=h \sin \left(x_{3} / \delta\right)$, with $\delta>0$. It is natural to assume that the amplitude of $f_{2}$ decreases with $\delta$. We conclude, assuming $\phi_{2}(0, x)=0$, that

$$
\varphi_{2}\left(t, x_{3}\right)=t \exp (t) f_{2}\left(x_{3}\right)
$$

and for the discussion, we assume $\varphi_{3}=0$. Next we approximate the $\omega_{1}$-vorticity equation for $x_{2}$ small and $x_{1} \geq \bar{x}_{1}>0$ with $\bar{x}_{1}$ small, by

$$
\dot{\omega}_{1}+x_{1} \frac{\partial \omega_{1}}{\partial x_{1}}-\omega_{1}=0
$$

with the "inflow boundary condition"

$$
\omega_{1}\left(\bar{x}_{1}, x_{2}, x_{3}\right)=\frac{\partial v_{2}}{\partial x_{3}}=t \exp (t) \frac{\partial f_{2}}{\partial x_{3}} .
$$


The equation for $\omega_{1}$ thus exhibits exponential growth, which is combined with exponential growth of the "inflow condition". Altogether we expect $\exp (t)$ perturbation growth of residual perturbations of size $h$, resulting in a global change of the flow after time $T \sim \log (1 / h)$, which can be traced in the computations.

We thus understand that the formation of streamwise streaks as the result of a force perturbation oscillating in the $x_{3}$ direction, which in the retardation of the flow in the $x_{2}$-direction creates exponentially increasing vorticity in the $x_{1}$-direction, which acts as inflow to the $\omega_{1}$-vorticity equation with exponential growth by vortex stretching. Thus, there is exponential growth at rear separation in both the retardation in the $x_{2}$-direction and the accelleration in the $x_{1}$ direction. Note that since the perturbation is convected with the base flow, the absolute size of the growth is related to the length of time the perturbation stays in a zone of exponential growth. Since the combined exponential growth is independent of $\delta$, it follows that large-scale perturbations with large amplitude have largest growth, which is also seen in computations with $\delta$ the distance between streamwise streaks as seen in Fig. 20.2 which does not seem to decrease with decreasing $h$.

Notice that at forward separation the retardation does not come form opposing flows, and the zone of exponential growth of $\omega_{2}$ is short, resulting in much smaller perturbation growth than at rear separation.

\section{Summary}

We have detected blowup of smooth potential flow into non-smooth turbulent flow in computed EG2 solutions. We have found by duality-based a posteriori error estimation that mean-value outputs of EG2 solutions are wellposed to tolerances of interest, showing that EG2 solutions are representative viscosity solutions of the Euler equations. We have given evidence that correct blowup detection is possible with finite mesh size. Altogether, we have presented evidence of (II).

\section{Acknowledgement.}

The first author would like to acknowledge financial support from the Swedish Foundation of Strategic Research.

\section{REFERENCES}

1. J.T. Beale, T. Kato and A. Majda, Remarks on the breakdown of smooth solutions of the Euler equations, Commun. Math. Phys. 94, 1984.

2. J.L. Bona, W.G. Pritchard and L.R. Scott, A posteriori error estimates for exact and approximate solutions of time-dependent problems, In Seminar on Numerical Analysis and Its Applications to Continuum Physics, Colecao ATAS 12, Sociedade Brasileira de Matematica, 102-111, 1980.

3. Garret Birkhoff, Hydrodynamics: a study in logic, fact and similitude, Princeton University Press, 1950. 
4. Sergei Chernyshenko, Peter Constantin, James Robinson and Endriss Titi, A posteriori regularity of the three-dimensional Navier-Stokes equations from computations.

5. Peter Constantin, Euler equations, Navier-Stokes Equations and Turbulence, CIME Lecture Notes, 2003.

6. Peter Constantin, On the Euler Equations of Incompressible Fluids, Bull. Amer. math. Soc. 44 (2007), 603-21.

7. Charles Fefferman, Existence and Smoothness of the Navier-Stokes Equations, Official Clay Mathematics Institute Millenium Problem for the Navier-Stokes equations.

8. Eduard Feireisl, Dynmaics of Viscous Compressible Fluids, Oxford University Press, 2004.

9. Jacques Hadamard, Sur les problmes aux dérivées partielles et leur signification physique. Princeton University Bulletin, 49-52, 1902.

10. J. Deng, T. Y. Hou, and X. Yu, Geometric properties and the non-blowup of the three-dimensional Euler equation, Comm. PDEs, 30:1 (2005), 225-243.

11. J. Deng, T. Y. Hou, and X. Yu, Improved Geometric Conditions for Nonblowup of the 3D Incompressible Euler Equation, Communication in Partial Differential Equations, 31 (2006), 293-306.

12. T. Y. Hou and R. Li, Dynamic Depletion of Vortex Stretching and NonBlowup of the 3-D Incompressible Euler Equations, J. Nonlinear Science, 16 (2006), 639-664.

13. J. Hoffman and C. Johnson, Computational Turbulent Incompressible Flow, Springer, 2007.

14. J. Hoffman and C. Johnson, Computational Thermodynamics, Springer, 2008.

15. J. Hoffman and C. Johnson, Resolution of d'Alembert's paradox, to appear in Journal of Mathematical Fluid Mechanics.

16. P. Moin and J. Kim, Tackling turbulence by supercomputers, Scientific American, 1997.

17. G. Ponce, Remarks on a paper by J.T. Beale, T. Kato and A. Majda, Commun. Math. Phys. 98, 349-352, 1985.

18. J. Stoker, Bull. Amer. Math. Soc. Am. Math., Vol 57(6), pp 497-99. 


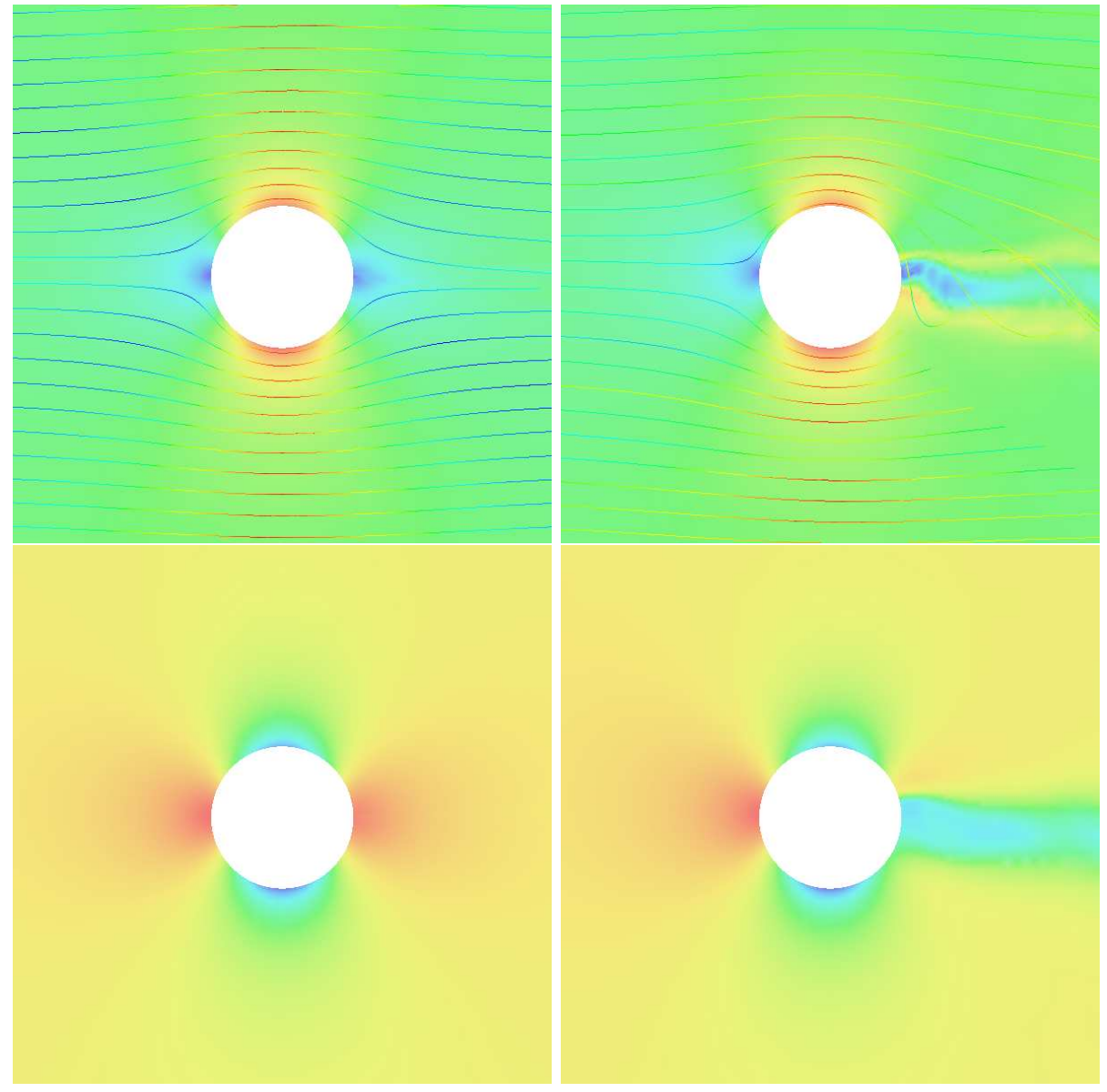

Figure 20.1: Flow past a cylinder; velocity (top) and pressure (bottom) for potential solution (left) and and EG2 turbulent solution (right). Notice the fully symmetric potential flow with zero drag, a and the low pressure wake from strong streamwise vorticity of the turbulent solution generating drag. 


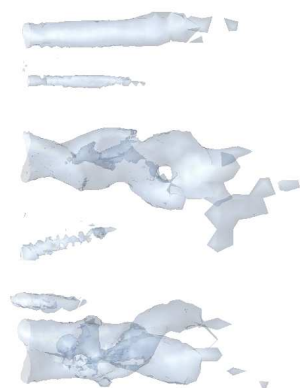

=000

4

एक
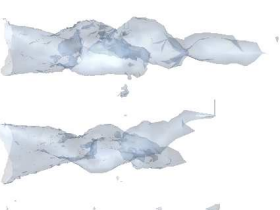

$+\infty$
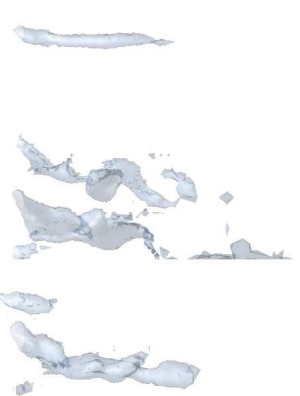

pr

$$
=
$$
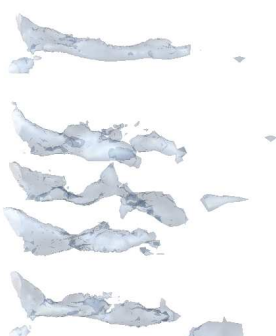
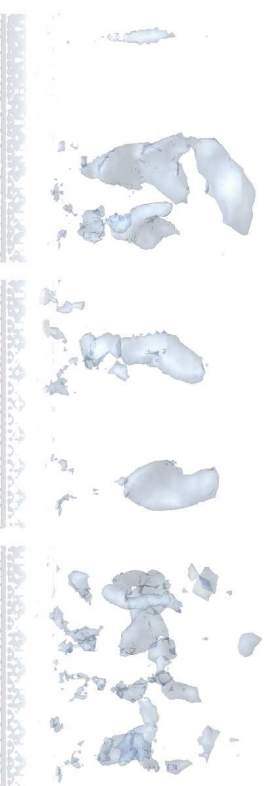

Figure 20.2: Levels surfaces of strong vorticity in EG2 solution: streamwise $\left|\omega_{1}\right|$ (left) and transversal $\left|\omega_{2}\right|$ (middle) and $\left|\omega_{3}\right|$ (right), at three times $t_{1}<t_{2}<t_{3}$ (upper, middle, lower), in the $x_{1} x_{3}$-plane.

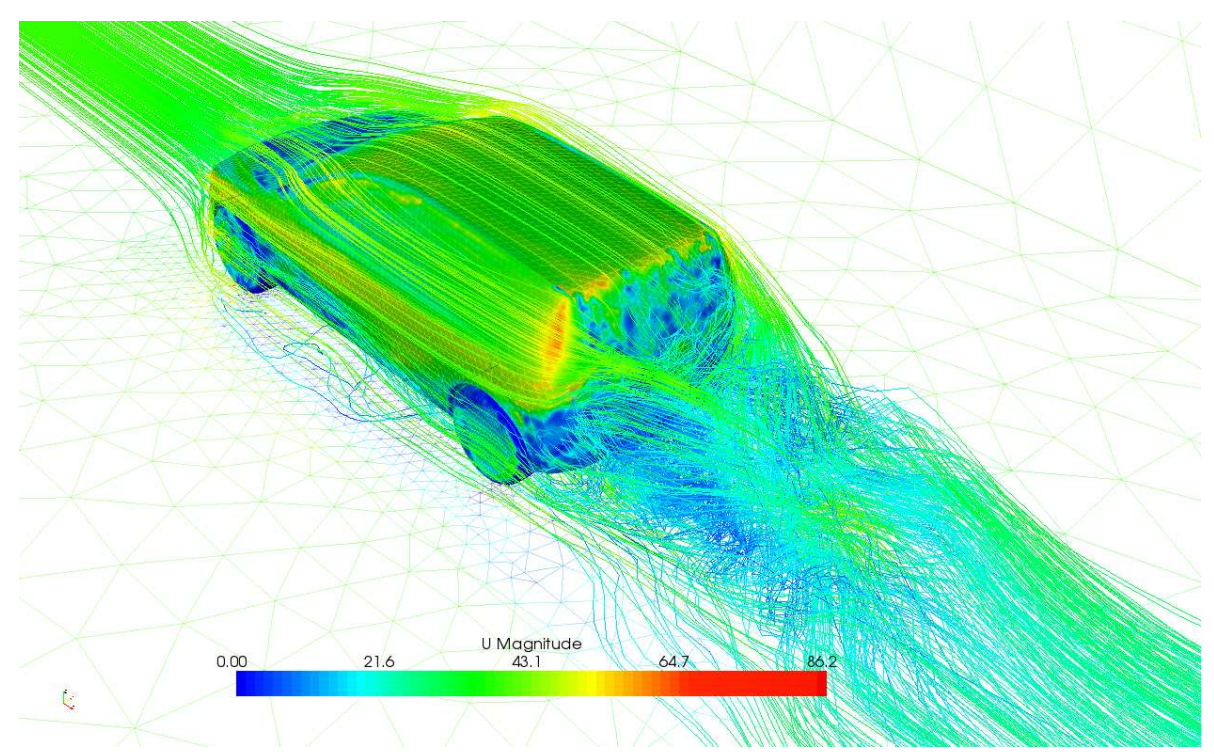

Figure 20.3: Streamlines and magnitude of tangential velocity for EG2 solution for flow around a car with turbulent wake and correct (substantial) drag (courtesy of Volvo Car for the geometry). 